\title{
MIXED SELFING AND RANDOM MATING WHEN HOMOZYGOTES ARE AT A DISADVANTAGE
}

\author{
B. I. HAYMAN \\ Agricultural Research Council's Unit of Biometrical Genetics, \\ Department of Genetics, University of Birmingham
}

Received 26.ix.52

\section{INTRODUCTION}

THIs investigation arose as part of a general investigation (Hayman and Mather, I953) of behaviour under inbreeding when homozygotes are at a selective disadvantage compared with heterozygotes. The inbreeding systems there discussed were handled by matrix methods not applicable to mixed selfing and random mating, for which an elementary algebraic treatment is necessary. This mating system occurs in plants which have an imperfect selfing mechanism combined with either widespread pollen dispersal or seed mixing by an external agency (as in cultivated crops).

One gene with only two alleles, $a$ and $b$, is considered, so that a population containing this gene may be classified into the three genotypes $a a, b b$, and $a b$ with frequencies $p, q$ and $r$. Any such population may be represented by a point in a trilinear diagram, based on an equilateral reference triangle, and such that the lengths of the perpendiculars from the "population point" to the sides of the triangle are proportional to $p, q$ and $r$. We shall investigate how a large population changes from generation to generation under certain conditions, and represent these changes by curves in a trilinear diagram. Further, we shall obtain phase diagrams, similar to fig. 4 of the paper quoted, showing broadly the equilibrium populations for various magnitudes of the selective disadvantages of the homozygotes $a a$ and $b b$. The populations are assumed to be large enough for random fluctuations in gene frequencies to be unimportant.

\section{EQUILIBRIUM}

Let $p, q$ and $r(p+q+r=\mathrm{I})$ be the frequencies of $a a, b b$ and $a b$ respectively and let $x, y$ and $\mathrm{I}(x$ and $y \leqslant \mathrm{I})$ be their respective viabilities. Let $s$ and $t(s+t=\mathrm{r})$ be the frequencies of selfing and random mating.

Starting with a population $\left(p_{0}, q_{0}, r_{0}\right)$ and no selection, the frequencies in the next generation under selfing are

$$
\left\{p_{0}+\frac{1}{4} r_{0}, q_{0}+\frac{1}{4} r_{0}, \frac{1}{2} r_{0}\right\}
$$

while under random mating they are

$$
\left\{\left(p_{0}+\frac{1}{2} r_{0}\right)^{2},\left(q_{0}+\frac{1}{2} r_{0}\right)^{2}, 2\left(p_{0}+\frac{1}{2} r_{0}\right)\left(q_{0}+\frac{1}{2} r_{0}\right)\right\}
$$


Under the mixed system and in the presence of selection they become $\left(p_{1}, q_{1}, r_{1}\right)$ where

$$
\begin{aligned}
p_{1} \propto x\left\{s\left(p_{0}+\frac{1}{4} r_{0}\right)+t\left(p_{0}+\frac{1}{2} r_{0}\right)^{2}\right\} & =x\left\{p_{0}+\frac{1}{4}\left(s r_{0}-t e_{0}\right)\right\} \\
q_{1} \propto y\left\{s\left(q_{0}+\frac{1}{4} r_{0}\right)+t\left(q_{0}+\frac{1}{2} r_{0}\right)^{2}\right\} & =y\left\{q_{0}+\frac{1}{4}\left(s r_{0}-t e_{0}\right)\right\} \\
r_{1} \propto \frac{1}{2} s r_{0}+2 t\left(p_{0}+\frac{1}{2} r_{0}\right)\left(q_{0}+\frac{1}{2} r_{0}\right) & =r_{0}-\frac{1}{2}\left(s r_{0}-t e_{0}\right) \\
\text { and } \quad e & =4 p q-r^{2}
\end{aligned}
$$
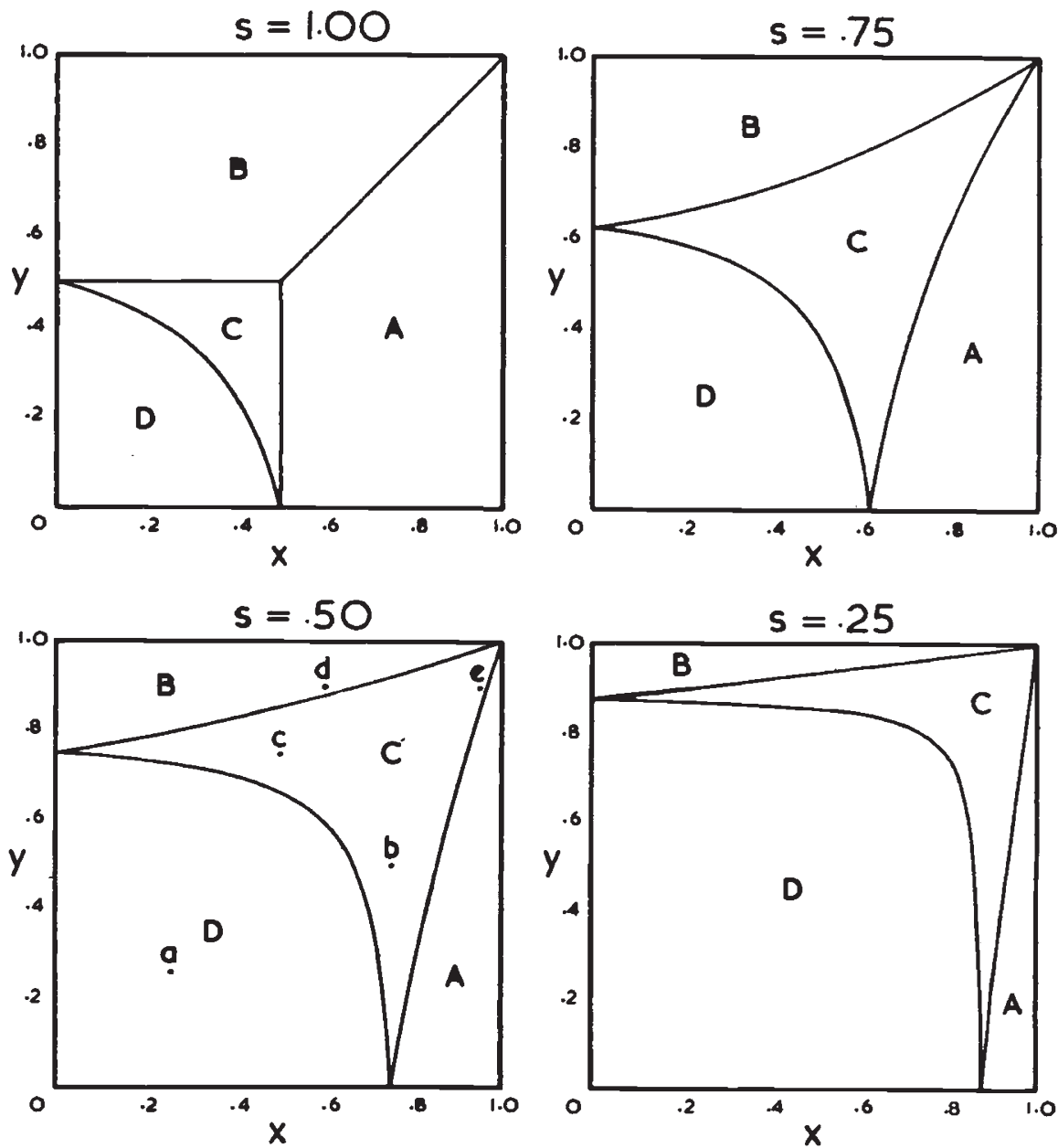

FIG. I.-Phase diagram showing types of equilibrium with various proportions of selfing $(s)$ and random mating $(t=1-s)$.

$x=$ survival rate of one homozygote $(a a)$ relative to that of the heterozygote $(a b)$. $y=$ relative survival rate of the other homozygote $(b b)$.

When the point $(x, y)$ is in A, the population becomes homozygous $a a$, in $\mathrm{B}$, homozygous $b b$. In $\mathrm{C}$, the population becomes less heterozygous than a population mating at random without selection, in $\mathrm{D}$, more heterozygous.

The points $a, b, c, d$ and $e$ in the diagram for $s=0.50$ are those referred to in 4.2 and fig. 4 . 
The population is in equilibrium if $\frac{p_{1}}{p_{0}}=\frac{q_{1}}{q_{0}}=\frac{r_{1}}{r_{0}}$, i.e. when

$$
x\left\{\mathrm{I}+\frac{\mathrm{I}}{4 p}(s r-t e)\right\}=y\left\{\mathrm{I}+\frac{\mathrm{I}}{4 q}(s r-t e)\right\}=\mathrm{I}-\frac{\mathrm{I}}{2 r}(s r-t e)
$$

These equations determine $p, q$ and $r$ in terms of $x, y$ and $s$.

Fig. I shows the type of equilibrium population for various values of $x, y$ and $s$. The areas marked $\mathrm{A}$ and $\mathrm{B}$ correspond to homozygons $a a$ and $b b$ respectively; in $\mathrm{G}$ the population, though containing heterozygotes, has more homozygotes than a population mating purely at random without selection; in $\mathrm{D}$ it contains more heterozygotes ; on the boundary between $\mathrm{C}$ and $\mathrm{D}$, the effects of inbreeding and selection exactly counterbalance each other. (The small letters in the diagram for $s=\frac{1}{2}$ will be referred to in 4.2.)

The inner boundaries of $\mathrm{A}$ and $\mathrm{B}$ are obtained by making $r$ tend to zero in the equilibrium equations (2). The boundary between $\mathrm{A}$ and $\mathrm{G}$ is

$$
2 s x y-2 x^{2}+2 x-s x-s y=0 .
$$

Interchanging $x$ and $y$ gives the boundary between $\mathrm{B}$ and $\mathrm{C}$.

Hardy's law is the statement that $e=0$. Eliminating $p, q$ and $r$ from this and equations (2) produces

$$
(2+s) x y-2 x-2 y+2-s=0 \text {. }
$$

This is the boundary between $\mathrm{G}$ and D. All three of these curves are hyperbolas.

A point to note is that strong selection against (even lethality of) one homozygote does not necessarily cause the corresponding allele to disappear from the population. Selection against the other homozygote can hold the heterozygote in the population.

\section{EXACT SOLUTIONS}

3.I. When $x=y=\mathrm{I}$, equations (2) show that the equilibrium state satisfies $s r-t e=0$. This is a parabola in the $(p, q, r)$ trilinear diagram passing through the lower vertices of the reference triangle. Fig. 2 contains equilibrium parabolas for $s=0, \frac{1}{2}, \frac{3}{4}$ and $\mathrm{I}$.

3.2. When $x=y<\mathrm{I}$, then from (2) $p=q$, and therefore

$$
e=(2 p-r)(2 p+r)=2 p-r \text {. }
$$

Eliminating $p, q$ and $t$ from (2) in this case leaves

$$
x r(\mathrm{I}+s-s r)=(\mathrm{I}-r)(\mathrm{I}-s+s r)
$$

giving the frequency, $r$, of the heterozygote in terms of the survival rate, $x$, of the homozygotes. Graphs of $r$ against $x$ for $s=0, \frac{1}{4}, \frac{1}{2}, \frac{3}{4}$ and $I$ are shown in fig. 3 .

In the trilinear diagram the equilibrium point is

$$
\left(\frac{1}{2}(\mathrm{I}-r), \frac{1}{2}(\mathrm{I}-r), r\right)
$$

on the perpendicular bisector of the base of the reference triangle. 
3.3. General values of $x$ and $y$. Eliminating $s r-t e$ from equations (2) leaves

$$
\text { where } \quad \begin{aligned}
a q(r+2 p) & =p(r+2 q) . \quad . \quad . \\
a y(\mathrm{I}-x) & =x(\mathrm{r}-y) .
\end{aligned}
$$

Equations (3) are, for various values of $a$, a set of hyperbolas through the vertices of the reference triangle.

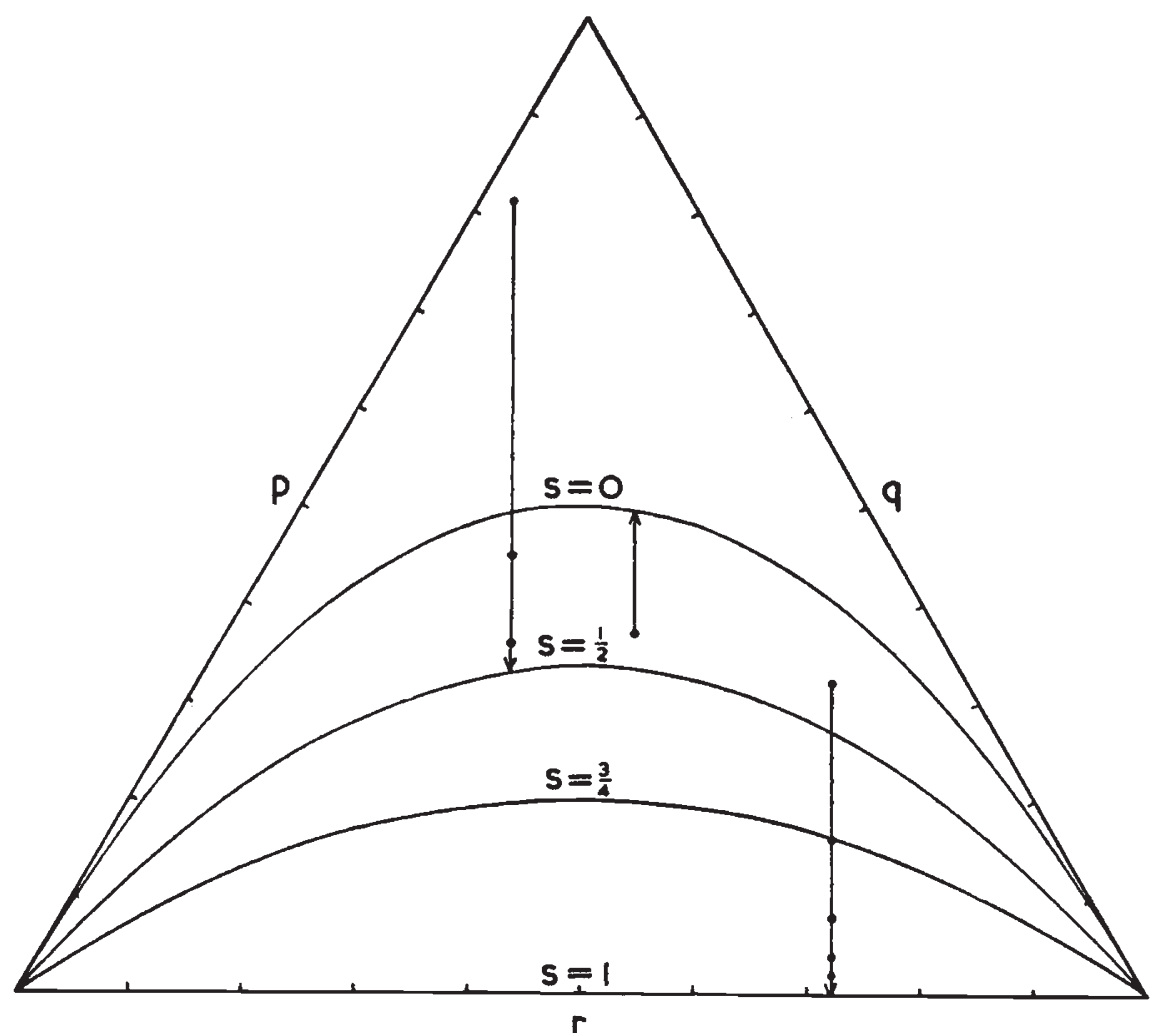

Fig. 2.- Trilinear diagram showing approach to equilibrium in the absence of selection. Distances from the sides labelled $p, q$ and $r$ represent the proportions of $a a, b b$ and $a b$ respectively in the population. $s$ is the proportion of selfing.

The points of the parabolas represent possible equilibrium populations for the corresponding values of $s$. The approach to equilibrium is shown for three cases by arrowed lines, the dots indicating the position of the population in successive generations.

Now there will be an equilibrium parabola through each equilibrium point. If it has equation

$$
(\mathrm{I}-\beta) r-\beta e=\mathrm{o}
$$

it must be satisfied by the solution of (2). The value of $\beta$ for this to be so can be shown to be the positive root of

$$
\beta^{2}(2 x y-x-y)+\beta\{(4-2 t) x y-(3-t)(x+y)+2\}+t(2-x-y)=0
$$


Once $a$ and $\beta$ have been evaluated, we can solve (3) and (4) for the equilibrium point. The solutions are

\begin{tabular}{|c|c|c|c|}
\hline & $\mathrm{C}, \mathrm{D}$ & $\mathrm{A}$ & $\mathrm{B}$ \\
\hline$p=$ & $d a\{\beta(\mathrm{I}+a)-\mathrm{I}+a\}$ & 1 & 0 \\
$q=$ & $d\{\beta(\mathrm{I}+a)+\mathrm{I}-a\}$ & 0 & 1 \\
$r=$ & $d\left\{\beta^{2}(\mathrm{I}+a)^{2}-(\mathrm{I}-a)^{2}\right\}$ & 0 & 0 \\
\hline
\end{tabular}

with $d^{-1}=\beta(\mathrm{I}+\beta)(\mathrm{I}+\alpha)^{2}$. The appropriate solution is that corresponding to the area (A, B, C or D) in which $(x, y)$ lies in fig. $\mathrm{I}$. When

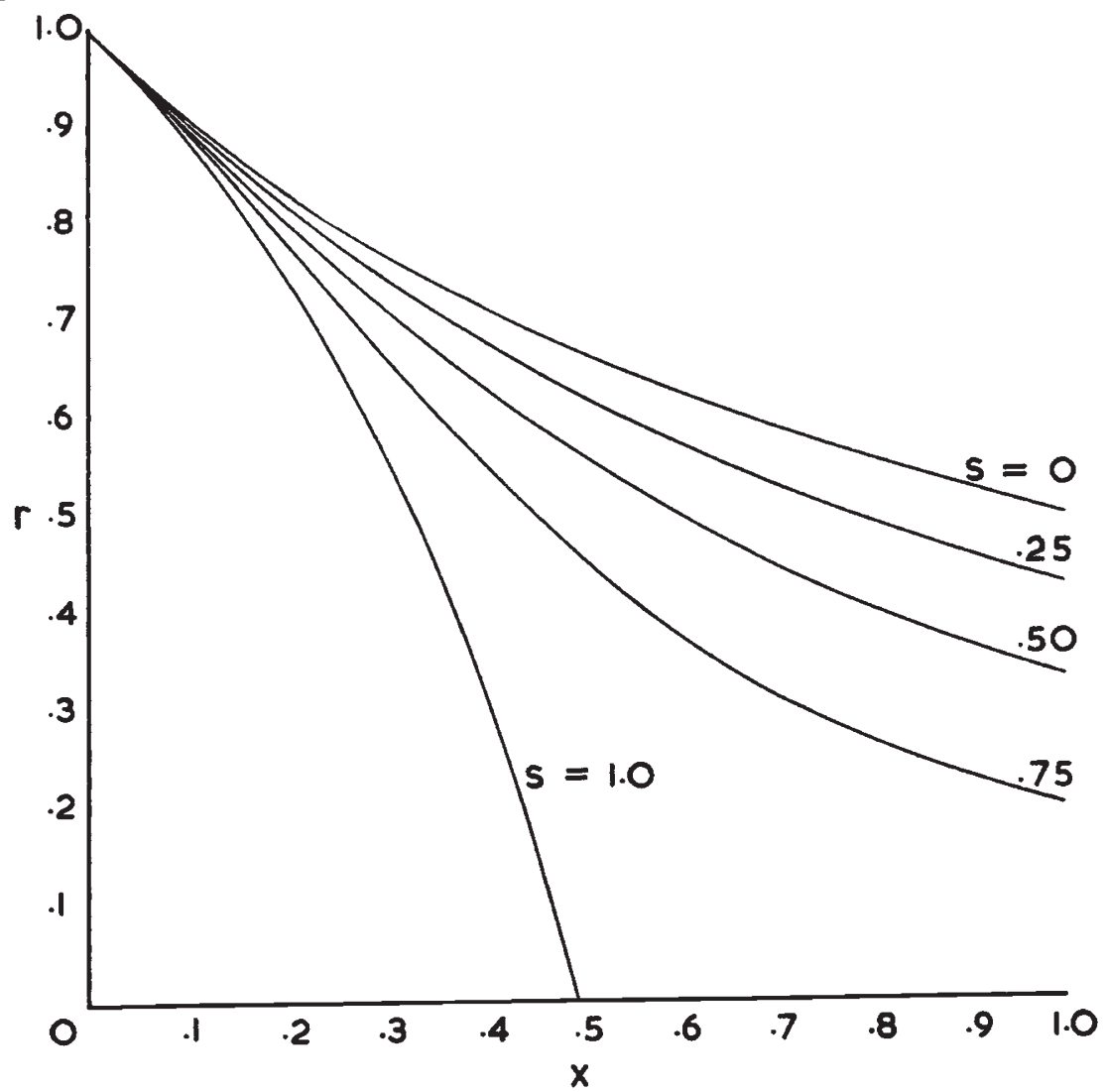

Fig. 3.-Equilibrium with equal viabilities of the homozygotes.

$r=$ proportion of heterozygotes in the population.

$x=$ survival rate of both homozygotes compared with the heterozygote.

$s=$ proportion of selfing.

$s$ is given and $(x, y)$ lies in $\mathrm{C}$ or $\mathrm{D}$ the equilibrium point lies above the parabola $s r-t e=0$, since the population must eventually become more heterozygous than it would in the absence of selection against the homozygotes. 
Evidently when $x$ and $y$ are less than unity, equilibrium is independent of the initial state (unless one allele is initially absent) and is determined by the two parameters $\alpha$ and $\beta$. As these are functions of the three population parameters $x, y$ and $s$, it is clear that different sets of values of $x, y$ and $s$ can determine the same pair of values of $\alpha$ and $\beta$, and hence the same equilibrium. This means, for example, that an increase in the proportion of selfing can be exactly counterbalanced by a decrease in the viability of the homozygotes. The diagrams of fig. I are sections for given $s$ of a 3 -dimensional graph of $x, y$ and $s$, and in this graph each line of intersection of a pair of surfaces, given by equations (3) and (4) determines one equilibrium point.

\section{THE APPROACH TO EQUILIBRIUM}

4.I. When $x=y=\mathrm{I}$, the statements (I) lead to the well-known results for the case of no selection. The proportionalities now become equations and further $s r_{1}-t e_{1}=\frac{1}{2} s\left(s r_{0}-t e_{0}\right)$

$$
\begin{aligned}
\therefore p_{n} & =p_{0}+\frac{1}{2} m_{n} \rightarrow p_{0}+\frac{1}{2} m=u^{2}+s u v /(2-s) \\
q_{n} & =q_{0}+\frac{1}{2} m_{n} \rightarrow q_{0}+\frac{1}{2} m=v^{2}+s u v /(2-s) \\
r_{n} & =r_{0}-m_{n} \rightarrow r_{0}-m=2 u v(2-2 s) /(2-s)
\end{aligned}
$$

where

$$
m_{n}=\left(s r_{0}-t e_{0}\right)\left(\mathrm{I}-\left(\frac{1}{2} s\right)^{n}\right) /(2-s)
$$

and

$$
m=\left(s r_{0}-t e_{0}\right) /(2-s)
$$

$m$ determines the limiting frequencies at equilibrium. The second form for the limiting frequencies indicates departures from a random mating population since $u=p_{0}+\frac{1}{2} r_{0}$ and $v=q_{0}+\frac{1}{2} r_{0}$, which are the gene frequencies.

In 3.I it was shown that the equilibrium point lies on the parabola $s r-t e=0$ in the trilinear diagram. Now $p_{n}+\frac{1}{2} r_{n}=p_{0}+\frac{1}{2} r_{0}$ and $q_{n}+\frac{1}{2} r_{n}=q_{0}+\frac{1}{2} r_{0}$, which also follow directly from the constancy of gene frequencies (assuming no mutation). Hence the point $\left(p_{n}, q_{n}, r_{n}\right)$ lies on the perpendicular line from $\left(p_{0}, q_{0}, r_{0}\right)$ to the base of the reference triangle. Evidently as $n$ increases the population point proceeds along this line until it reaches the equilibrium parabola, the distance from equilibrium being reduced to $\frac{1}{2} s$ of its previous value every generation. Fig. 2 illustrates three cases when $s=0$, $\frac{1}{2}$ and $\mathrm{I}$, the first attaining equilibrium in one generation. The dots indicate successive population points.

4.2. For general values of $x$ and $y$, successive applications of the proportionalities ( $\mathrm{I}$ ) enable the population point to be plotted from generation to generation, while the limit point may be determined independently by 3.3. Fig. 4 illustrates the approaches to equilibrium starting with two different populations $F=(0.6,0 \cdot 3,0 \cdot 1)$ or $\mathrm{G}=(0 . \mathrm{I}, 0.3,0.6)$ under an equal mixture of selfing and random mating $\left(s=\frac{1}{2}\right)$. Commencing at $\mathrm{F}$, the first few and some subsequent 
generations have been indicated by numbered dots. Five sets of selection values $(x, y)$ giving five equilibrium points have been chosen as in the table.

\begin{tabular}{|c|c|c|c|c|c|}
\hline & \multicolumn{5}{|c|}{ Equilibrium points } \\
\hline & $a$ & $b$ & $c$ & $d$ & $e$ \\
\hline $\begin{array}{rrr}\text { Selection values } x & . & . \\
y & . & .\end{array}$ & $\begin{array}{l}0 \cdot 259 \\
0 \cdot 259\end{array}$ & $\begin{array}{l}0.750 \\
0.500\end{array}$ & $\begin{array}{l}0.500 \\
0.750\end{array}$ & $\begin{array}{l}0 \cdot 600 \\
0 \cdot 900\end{array}$ & $\begin{array}{l}0.950 \\
0.900\end{array}$ \\
\hline $\begin{array}{r}\text { Equilibrium frequencies } p \text {. } \\
\qquad \begin{array}{r}r \\
r\end{array} .\end{array}$ & $\begin{array}{l}0 \cdot 125 \\
0 \cdot 125 \\
0 \cdot 75^{0}\end{array}$ & $\begin{array}{l}0 \cdot 634 \\
0 \cdot 057 \\
0 \cdot 309\end{array}$ & $\begin{array}{l}0.057 \\
0.634 \\
0.309\end{array}$ & $\begin{array}{l}0 \cdot 000 \\
1 \cdot 000 \\
0 \cdot 000\end{array}$ & $\begin{array}{l}0.724 \\
0 \cdot 072 \\
0.204\end{array}$ \\
\hline
\end{tabular}

These have also been labelled in fig. I for $s=\frac{1}{2}$.

Some points may be noted. Most of the initial disequilibrium

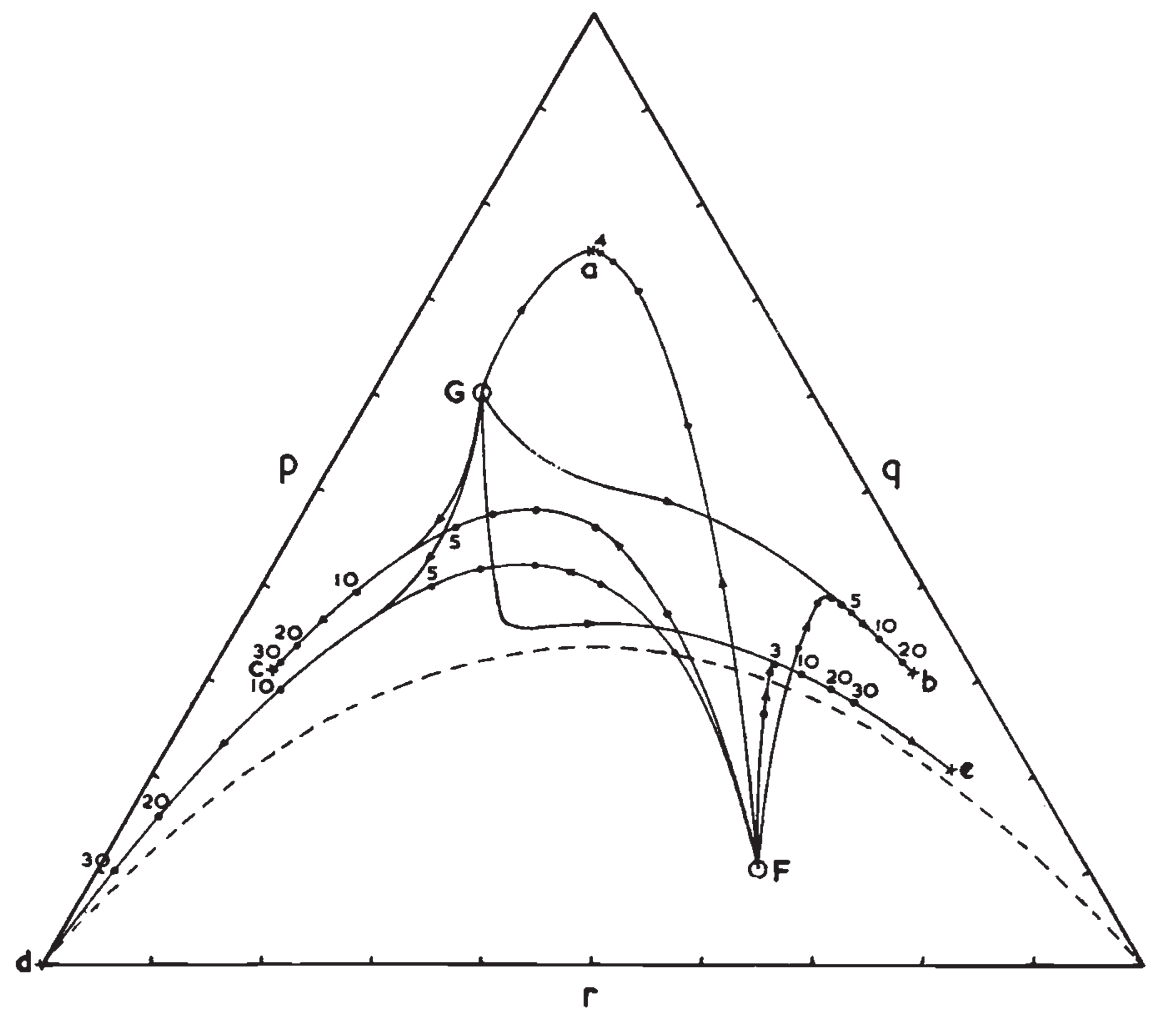

FIg. 4.-Trilinear diagram of the approach to equilibrium when the proportion of selfing, $s=\frac{1}{2}$.

$\mathrm{F}$ and $\mathrm{G}$ are two possible starting points while $a, b, c, d$, and $e$ are five equilibrium points corresponding to the values of $(x, y)$ shown in fig. $\mathrm{I}, s=\frac{1}{2}$. The solid lines show the paths of the population from $\mathrm{F}$ or $\mathrm{G}$ to $a, b, c, d$ or $e$. The dots on the lines from $F$ show the position of the population after the number of generations indicated.

The broken line represents possible equilibria in the absence of selection. 
between heterozygotes and homozygotes is rapidly removed : the disequilibrium between the two homozygotes disappears very slowly.

With strong selection against the homozygotes the equilibrium is attained quickly and fairly directly. With weak selection against the homozygotes, the equilibrium parabola through the equilibrium point is first approached quickly and nearly vertically, thus correcting any heterozygote-homozygote disequilibrium. Then the population point moves slowly round close to this parabola to the final equilibrium point, thus correcting any disequilibrium between the two homozygotes.

We recall that when there is no selection against the homozygotes the equilibrium depends on the initial gene frequencies. Hence, if fluctuations in these frequencies occur (by chance or by mutation) the equilibrium point shifts around the equilibrium parabola (determined by $s$ ) since there is no controlling force to hold it at any particular point on the parabola.

When there is only weak selection against the homozygotes a similar situation prevails. In case $(e)$ and starting from $(0 \cdot 6,0 \cdot 3,0 \cdot 1)$ the population point is still distant 0.125 units from the equilibrium point after 30 generations and the next generation only reduces this gap by $2 \cdot 1$ per cent. This slow approach to equilibrium shows that the force working towards equilibrium is weak. This means that, though in general the equilibrium is stable and is fixed by the mating system and the survival rate of the homozygotes, there is a region near $x=y=\mathrm{I}$ in fig. $\mathrm{I}$ in which small disturbing influences may cause the population point to drift widely away from equilibrium along the equilibrium parabola.

\section{SUMMARY}

The equilibrium is investigated of a large population breeding under a system of mixed selfing and random mating with selection against the homozygotes. Diagrams are produced giving the types of equilibrium and illustrating the approach to equilibrium in particular cases.

\section{REFERENCES}

HAYMAN, B. I., AND MATHER, K. 1953. The progress of inbreeding when homozygotes are at a disadvantage. Heredity, $7,165_{-183}$. 